\section{Harmonics in the Piezo-Electric Oscillation of a Quartz Crystal}

S. Parthasarathy, A. Pande and M. Pancholy ${ }^{1}$ have recently given evidence of oscillation of the quartz crystal not only at odd harmonics but also at even and half-odd harmonics. In the third column of Table 1 in their communication, there are fourteen values of the ratio of the frequency to the fundamental which they got from a single quartz plate : $0 \cdot 499,1 \cdot 00,1 \cdot 465,1 \cdot 96,2 \cdot 69,3 \cdot 00,3 \cdot 50,4 \cdot 00,4 \cdot 40$, $5 \cdot 00,5 \cdot 35,6 \cdot 23,7 \cdot 04$, and $8 \cdot 00$.

In the course of an experiment on supersonic waves propagated in liquid, we have also noticed the harmonics produced by the piezo-electric oscillation of a quartz crystal on the background of a continuous range of frequencies of oscillation. The electric circuit we used was an ordinary Hartley type with an output of about 6 watts only. Eleven quartz plates, of different shapes, circular, rectangular and square, were used. They were cut and ground in this Institute, except one which was given to us in exchange by the American Air Force in Kunming. They were either $A T$-cut or $X$-cut, and no thickness variation on each plate more than $0.005 \mathrm{~mm}$. was observed.

The quartz plate was clamped on a holder immersed in distilled water. A hot-cathode sodium discharge lamp of 40 watts was used as light source. The diffraction pattern was observed with a reading telescope of focal length $247 \mathrm{~mm}$. The frequency of oscillation was measured with a $G$. E. wave-meter of precision type.

As soon as the electric circuit began to oscillate, the diffraction pattern appeared; as the frequency of the electric circuit increased by turning the condenser in the tank circuit, the spacing between the diffraction lines was gradually and continuously widened. The diffraction pattern usually gave one or two orders. However, at certain particular frequencies, the milliammeter in the plate circuit recorded a sudden jump; at the same time the diffraction pattern went up to a higher order and its intensity abruptly increased. For example, one of eleven quartz plates was a circular disk of $18 \mathrm{~mm}$. in diameter and $1.337 \mathrm{~mm}$. in thickness. It was $X$-cut, and its fundamental frequency measured 2,080 kc. It was then submitted to the electric circuit for oscillation. From 2,000 to $14,000 \mathrm{kc}$. there were twelve frequencies where the jumps on the milliammeter occurred and the order and intensity of the diffraction pattern increased. The exact values of the ratio of the frequency to the fundamental were found to be $1 \cdot 00,1 \cdot 72,1 \cdot 94,2 \cdot 04,2 \cdot 16,2 \cdot 65,3 \cdot 00$, $3 \cdot 13,4 \cdot 00,4 \cdot 45,5 \cdot 00,6 \cdot 20$. They were believed to be closely, if not exactly, the odd, even and half-odd harmonics which Parthasarathy and his collaborators found. Throughout the whole range of oscillation, the diffraction patterns never failed to appear, although at certain places they were barely visible even with the help of a telescope.

It will be remembered that the Hartley circuit has a strong feed-back, so the crystal is forced to vibrate. It seems that the quartz crystal can be forced to vibrate over a wide range of frequency as soon as its impedance has been overcome, and that it particularly shows the resonance of vibration at the frequencies of $\frac{p+1}{2} f$, where $f$ is the fundemental frequency of the quartz crystal and $p$ is an integer. The odd har- monics oscillate much more strongly than the even. as is indicated by the intensity and number of orders of the diffraction pattern. If we put the crystal in a Pierce circuit which has a weak feed-back and is generally regarded as less active in oscillation, the crystal only oscillates at its fundamental frequency and odd harmonics. It may be added that in the forced vibration, the harmonic vibrations do not come out very exactly, and they may change their resonance frequencies when a different mode of excitation, as in different conditions of clamping the crystal, is used.

\section{National Academy of Peiping,} Institute of Physies, Kunming. May 1.

${ }^{1}$ Parthasarathy, S., Pande, A., and Pancholy, M., J. Sci. and Indust. Res., 2, No. 5, 295 (1944).

\section{Hexachlorocyclohexane as an Insecticide}

My attention has been directed to the possibility that Dr. L. B. Bourne's letter ${ }^{1}$ may be read as implying that Dr. E. L. Taylor's reference ${ }^{2}$ to hexachlorocyclohexane, supplied by Imperial Chemical Industries, as a "new" insecticide was unjustified in the light of recent information that this material has been available as an insecticide in France for some years.

I am asked to emphasize that it was in the spring of 1943 that I.C.I. made the important and unexpected discovery that the remarkable insecticidal properties of hexachlorocyclohexane are almost entirely associated with only one of the four isomers (gamma) which had first been isolited thirty-one years earlier. The Company, however, was not permitted by the Government to disclose this or the extent to which hexachlorocyclohexane had been manufactured and used as an insecticide in Britain until 1945, when an announcement was made in the Hurter Memorial Lecture (not yet published) to the Society of Chemical Industry on March 8. This discovery of the outstanding insecticidal activity of 'Gammexane', as the gamma isomer has been called, made possible a planned development of hexachlorocyclohexane as an insecticide.

Thus sufficient material was produced to meet the 1943 demands of agriculture for a dust to replace derris, then in short supply, for the control of flea beetle. The material proved so successful, not only in the control of many agricultural and horticultural pests, but also in trials against locusts and industrial pests such as cockroaches, crickets, bed bugs, flies, etc., that production was increased in the following two years and the material was made available for these widely varied applications, Up to the time when the Government permitted the disclosure of the nature of the active constituent of the material, hexachlorocyclohexane equivalent to several thousand tons of insecticidal dust had been produced in Britain. SIDNEY ROGERSON.

Imperial Chemical Industries, Limited, Nobel House,

2 Buckingham Gate, London, S.W.1.

1 Nature, 156, 85 (1945).

Nature, 165, 393 (1945). 